\title{
Comparison of outcome between intrauterine balloon tamponade and uterine artery embolization in the management of persistent postpartum hemorrhage: A propensity score- matched cohort study
}

\author{
Paul I. Ramler ${ }^{1,2}$ (D) | Dacia D. C. A. Henriquez ${ }^{1,2}$ (D) | Thomas van den Akker ${ }^{2,3,4}$ (D) | \\ Camila Caram-Deelder ${ }^{1,5}$ (D) \\ Rolf H. H. Groenwold ${ }^{5,6}$ (i) | Kitty W. M. Bloemenkamp \\ Jos van Roosmalen ${ }^{2,4}$ (D) | Jan M. M. van Lith ${ }^{2}$ | Johanna G. van der Bom ${ }^{1,5}$ iD | on behalf \\ of the TeMpOH-1 study group* \\ ${ }^{1}$ Center for Clinical Transfusion Research, Sanquin Research, Leiden, the Netherlands \\ ${ }^{2}$ Department of Obstetrics, Leiden University Medical Center, Leiden, the Netherlands \\ ${ }^{3}$ National Perinatal Epidemiology Unit (NPEU), University of Oxford, Oxford, UK \\ ${ }^{4}$ Faculty of Science, Athena Institute, VU University Amsterdam, Amsterdam, the Netherlands \\ ${ }^{5}$ Department of Clinical Epidemiology, Leiden University Medical Center, Leiden, the Netherlands \\ ${ }^{6}$ Department of Biomedical Data Sciences, Leiden University Medical Center, Leiden, the Netherlands \\ ${ }^{7}$ Department of Obstetrics, Birth Center Wilhelmina Children Hospital, Division Woman and Baby, University Medical Center Utrecht, Utrecht, the \\ Netherlands
}

\section{Correspondence}

Johanna G. van der Bom, Plesmanlaan 1a 5th floor, 2333 BZ Leiden, the Netherlands.

Email: j.g.van_der_bom@lumc.nl

Funding information

The study was supported by an internal grant from Sanquin Center for Clinical Transfusion Research (PPOC 11-023). Rolf H. H. Groenwold received funding from the Netherlands Organisation for Scientific Research (ZonMw, project 917.16.430). The funding bodies had no role in the design, conduct or decision to publish this study.

\begin{abstract}
Introduction: To compare the outcomes of women who were initially managed by intrauterine balloon tamponade or uterine artery embolization because of persistent postpartum hemorrhage demanding an immediate intervention to control bleeding. Material and methods: Propensity score-matched cohort study including women who had intrauterine balloon tamponade or uterine artery embolization as initial management strategy to control persistent postpartum hemorrhage, that is, refractory to first-line therapy combined with at least one uterotonic agent. The primary outcome measure was a composite of peripartum hysterectomy and/or maternal mortality. Secondary outcomes measures were total volume of blood loss and total number of packed red blood cells transfused.

Results: Our 1:1 propensity score-matched cohort comprised of 50 women who had intrauterine balloon tamponade and 50 women who underwent uterine artery embolization at a blood loss between 1000 and $7000 \mathrm{~mL}$. There was no statistically
\end{abstract}

Abbreviations: $\mathrm{Cl}$, confidence interval; IQR, interquartile range; IUBT, intrauterine balloon tamponade; MNM, maternal near miss; MWU, Mann-Whitney U; OR, odds ratio; PS propensity score; SMD, standardized mean difference; TeMpOH-1, Transfusion strategies in women during major obstetric haemorrhage; UAE, uterine artery embolization; WSR, Wilcoxon signed-rank.

*The complete membership of the TeMpOH-1 study group is provided in Appendix S1. This is an open access article under the terms of the Creative Commons Attribution-NonCommercial License, which permits use, distribution and reproduction in any medium, provided the original work is properly cited and is not used for commercial purposes.

(c) 2019 The Authors. Acta Obstetricia et Gynecologica Scandinavica published by John Wiley \& Sons Ltd on behalf of Nordic Federation of Societies of Obstetrics and Gynecology (NFOG) 
significant difference in the hysterectomy risk between the two groups ( $n=6$ in each group, odds ratio [OR] 1.00, 95\% confidence interval [Cl] .30-3.34), in total volume of blood loss (median $4500 \mathrm{~mL}$, interquartile range [IQR] 3600-5400) for balloon vs $4000 \mathrm{~mL}$ (IQR 3250-5000) for embolization, $P=0.382$ ) or in total units of packed red blood cells transfused (median 7 (IQR 5-10) for balloon vs 6 [IQR 4-9] for embolization, $P=0.319)$. Fifteen women (30\%) who were initially managed by an intrauterine balloon still underwent uterine artery embolization, of whom one had an embolization-related thrombo-embolic event. Maternal mortality occurred in neither of the intervention groups.

Conclusions: No difference in the risk of peripartum hysterectomy and/or maternal death was observed between women who had intrauterine balloon tamponade and women who underwent uterine artery embolization as an initial management for persistent postpartum hemorrhage. Although this study was underpowered to demonstrate equivalence, our study design provides a framework for future research in which intrauterine balloon tamponade may prove to be a suitable intervention of first choice in the management of persistent postpartum hemorrhage.

\section{KEYWORDS}

intrauterine balloon tamponade, maternal mortality, peripartum hysterectomy, postpartum hemorrhage, propensity score, severe maternal outcome, uterine artery embolization

\section{1 | INTRODUCTION}

Postpartum hemorrhage remains the leading cause of maternal mortality around the world. ${ }^{1}$ There is an international call for improving maternal safety and the evaluation of obstetric care is crucial to answer this call and reduce maternal deaths, which are often preventable. ${ }^{2}$ Peripartum hysterectomy can be performed as a life-saving procedure of last resort but leads to infertility, accompanied by substantial morbidity and psychosocial sequelae. ${ }^{3-5}$ Various invasive and less invasive management strategies were developed to reduce the need for hysterectomy after birth, including intrauterine balloon tamponade, uterine compression sutures and devascularization of the uterine artery by surgical ligation or radiological embolization. ${ }^{6}$

Uterine artery embolization may be used to manage persistent postpartum hemorrhage that demands immediate intervention before proceeding to hysterectomy but it is considered a relatively costly and invasive procedure that is prone to complications (eg, post-embolization syndrome, thrombo-embolic events or uterine necrosis). ${ }^{7-10}$ On the other hand, intrauterine balloon tamponade has emerged as an inexpensive and less invasive option to control ongoing bleeding. ${ }^{11-13}$ Insertion of an intrauterine balloon for the purpose of tamponade during postpartum hemorrhage could potentially obviate the need for uterine artery embolization, and reduce healthcare costs. However, these interventions have never been compared in terms of effectiveness of preventing severe maternal outcome (ie, maternal death or a near miss averted by a peripartum hysterectomy) and thus uncertainty persists as to whether intrauterine balloon tamponade is an effective alternative to

\section{Key message}

Intrauterine balloon tamponade during persistent postpartum hemorrhage could obviate the need for embolization in most women without an increased risk of severe maternal outcome.

uterine artery embolization when both interventions are considered as possible options during the course of postpartum hemorrhage.

The aim of this study was to compare severe maternal outcome in women who received intrauterine balloon tamponade with women who had uterine artery embolization as initial management for persistent postpartum hemorrhage in whom immediate intervention was deemed necessary.

\section{2 | MATERIAL AND METHODS}

Given that intrauterine tamponade with a balloon-like device is less invasive and much easier to perform than uterine artery embolization, it is possible that intrauterine balloon tamponade is more often used in women with less severe bleeding. Women receiving intrauterine balloon tamponade may also differ in various ways from women undergoing uterine artery embolization. For these reasons, we used propensity score-matching to correct for any confounding by indication. Using this technique, we constructed a cohort of women who 
differed with respect to the management strategy applied but were similar with respect to all other clinically relevant characteristics that could have influenced the clinician's decision to apply one of the interventions during persistent postpartum hemorrhage. ${ }^{14}$

\section{1 | Data source}

This study used data from the Transfusion strategies in women during Major Obstetric Haemorrhage study (TeMpOH-1). The $\mathrm{TeMpOH}-1$ study was a nationwide retrospective cohort study in 61 hospitals in the Netherlands (71\% of all hospitals in the country) in which data from medical files of pregnant women of at least 18 years old were included. These women had received at least four units of packed red blood cells or any transfusion of fresh frozen plasma and/or platelets in addition to packed red blood cells because of obstetric hemorrhage ( $\geq 1000 \mathrm{~mL}$ blood loss during pregnancy or the first 24 hours following birth) between 1 January 2011 and 1 January 2013. Eligible women were identified by cross-referencing data from hospital blood transfusion services with the local birth registers in participating hospitals. Trained medical students and research nurses obtained available data from medical records present in maternity units, operating theaters and intensive care units.

\subsection{Cohort selection}

From the TeMpOH-1 database, we identified all women who were initially managed by intrauterine balloon tamponade or uterine artery embolization during persistent postpartum hemorrhage. Persistent postpartum hemorrhage was defined as ongoing hemorrhage within the first 24 hours following birth, refractory to first-line therapy (previously defined per primary cause of hemorrhage, Table $\mathrm{S} 1)^{15}$ combined with the administration of at least one uterotonic agent (including oxytocin [prophylactic use of oxytocin following childbirth excluded], ergometrine, misoprostol or sulprostone). By using this definition of persistent postpartum hemorrhage, we avoided a definition solely based on mere estimation of blood loss and ensured that women included in this study received minimally necessary care per cause of hemorrhage prior to use of intrauterine balloon tamponade or uterine artery embolization. However, since no uterine artery embolizations were performed when there was $<1000 \mathrm{~mL}$ blood loss and no intrauterine balloons were inserted when there was $>7000 \mathrm{~mL}$ blood loss (Figure S1), we restricted our analyses to women who had intrauterine balloon tamponade or uterine artery embolization between these limits of blood loss. Furthermore, although the Bakriß balloon (Cook Medical, Bloomington, IN, USA) is the type of intrauterine balloon device mostly used in the Netherlands, the TeMpoH-1 study did not specifically register which type of device was inserted. Therefore, this study defined intrauterine balloon tamponade as insertion of any type of balloon catheter into the uterine cavity for the purpose of tamponade. Women were classified depending on the intervention (ie, balloon or embolization) that was first applied and they were considered to remain in that intervention group until end of hemorrhage or occurrence of the primary outcome.

\section{3 | Outcome measures}

The World Health Organization developed the Maternal Near Miss (MNM) tool to enable uniform identification of those women who nearly died but survived a complication during pregnancy, childbirth or within 42 days of termination of pregnancy. ${ }^{16}$ In this approach, women who underwent peripartum hysterectomy due to hemorrhage are considered MNM. The reason to perform uterine balloon tamponade or uterine artery embolization is to control intractable bleeding and to avert severe maternal outcome (ie, maternal death or MNM). Hence, we used a composite of maternal death or MNM averted by peripartum hysterectomy as the primary outcome measure. If this primary outcome did not occur, end of bleeding was defined as the time of the last estimated blood loss measurement. Secondary outcome measures were total estimated volume of blood loss and total number of packed red blood cells transfused.

\subsection{Statistical analyses}

The propensity score, representing the probability of receiving intrauterine balloon tamponade during the course of persistent postpartum hemorrhage, was estimated by a logistic regression model with intrauterine balloon tamponade inserted between an estimated blood loss of $1000-7000 \mathrm{~mL}$ as the dependent variable. Characteristics considered to be potential confounders for the association between use of intrauterine balloon tamponade or use of uterine artery embolization, or characteristics considered to be risk factors for the occurrence of the primary outcome measure alone, were included as covariates in the propensity score model. ${ }^{17}$

Characteristics included as covariates that were available at the moment the clinician decided to use intrauterine balloon tamponade or perform uterine artery embolization were: maternal age, gestational age, parity (nulliparity or multiparity), preeclampsia, multiple pregnancy, prior cesarean birth, mode of birth (vaginal birth or cesarean section), cause of hemorrhage (categorized as uterine atony, retained placenta, abnormally invasive placenta and other causes [composite of placenta previa, placental abruption and uterine rupture due to small numbers]), the presence of coagulopathy (defined as a fibrinogen level $\leq 2 \mathrm{~g} / \mathrm{L}$ during bleeding), symptoms of shock (defined as at least one measurement of a systolic blood pressure $\leq 90 \mathrm{~mm} \mathrm{Hg}$ and/or heart rate $\geq 120$ beats per minute during bleeding), volume of blood loss at time of intervention (measured by weighing gauzes or other soaked material and use of suction in the operating theatre), hemostatic interventions used at the time of intrauterine balloon tamponade or uterine artery embolization (the number of uterotonic agents given [including oxytocin, ergometrine, misoprostol and sulprostone], the administration of non-uterotonic agents [tranexamic acid, fibrinogen concentrate and recombinant factor $\mathrm{VIla}$, and number of packed red blood cells, fresh frozen plasma and platelets transfused), and other surgical interventions that had already been applied at the time of intrauterine balloon insertion or uterine artery embolization (composite of B-Lynch suture and uterine artery ligation). These clinically relevant characteristics 
were selected a priori based on the literature $e^{4,5,7,12,13,18-23}$ and clinical reasoning. Missing variables were imputed using median and logically derived imputation (see Appendix S2 for the rationale behind the imputation method applied per missing variable).

To balance all characteristics over the course of persistent postpartum hemorrhage, estimated blood loss was stratified into increments of $500 \mathrm{~mL}$ and women were matched within the same increment of blood loss in which they had the intervention. Thus, women who had intrauterine balloon tamponade during persistent postpartum hemorrhage were matched with women with the same chance (ie, same propensity score) of receiving intrauterine balloon tamponade but who underwent uterine artery embolization instead within the same increment of blood loss at the time of intervention. By matching in the same increments of blood loss, we ensured that women who had intrauterine balloon tamponade were matched with women who had uterine artery embolization with approximately the same amount of blood loss. Matching was performed by a 1:1 sequential greedy algorithm without replacement using a caliper of .2 times the standard deviation of the logit of the propensity score. ${ }^{24}$ Balance in distribution of clinically relevant characteristics between both groups was assessed by standardized differences, where distributions of characteristics were considered comparable when the standardized difference was $<10 \%$ after propensity score-matching. ${ }^{25,26}$ Interaction terms were included in the propensity score model if they improved balance between the comparison groups after propensity score-matching. ${ }^{27}$

The primary outcome was compared between women who were managed by intrauterine balloon tamponade and women who underwent uterine artery embolization using a logistic regression model, resulting in estimated odds ratios (OR) with $95 \%$ confidence intervals (CI). ${ }^{28}$ Differences in secondary outcome measures were estimated by Mann-Whitney $U$ testing before propensity score-matching, and by the Wilcoxon signed-rank test after propensity score-matching, where a two-tailed $P$ value $<.05$ was considered statistically significant. ${ }^{29}$ To evaluate the robustness of our study findings with regard to propensity score-matching, a sensitivity analysis was performed of the primary outcome measure by including the propensity score as a covariate in the logistic regression model to compare the primary outcome measure between both intervention groups, under the assumption that the propensity score has a linear functional relation with the log odds of the primary outcome. ${ }^{30}$

All continuous variables were summarized as medians with interquartile ranges (IQR), and categorical variables were presented as frequencies with percentages (\%). All statistical analyses were performed using the STATA STATISTICAL SOFTWARE: Release 14 (StataCorp LP, College Station, TX, USA). The statistical analysis plan was approved by the Scientific Committee of the Sanquin Center for Clinical Transfusion Research before execution of the analyses.

\subsection{Ethical approval}

The TeMpOH-1 study was approved by the Ethical Committee of Leiden University Medical Center (P12.273; 31 January 2013) and by the institutional review boards of all participating hospitals. The TeMpOH-1 study was registered in the Netherlands Trial Register (Trial NL3909; 17 July 2013) and need to obtain informed consent was waived by the ethics committee.

\section{3 | RESULTS}

Of the 270101 women who gave birth in the Netherlands during the 2-year inclusion period, 1391 women endured postpartum hemorrhage and fulfilled the inclusion criteria of the $\mathrm{TeMpOH}-1$ study, 1260 of whom had ongoing bleeding despite the first-line therapy combined with at least one uterotonic agent. We identified a total of 373 women who were initially managed by intrauterine balloon tamponade and 82 women who initially had uterine artery embolization at an estimated blood loss between 1000 and $7000 \mathrm{~mL}$ to control bleeding. Eleven balloons were inserted when there was less than $1000 \mathrm{~mL}$ blood loss and five women underwent uterine artery embolization when there was blood loss exceeding $7000 \mathrm{~mL}$. Of the 373 women who initially had intrauterine balloon tamponade, 50 were propensity score-matched with 50 of 82 women who initially underwent uterine artery embolization during persistent postpartum hemorrhage (Figure 1).

\section{1 | Comparison of characteristics}

Clinically relevant characteristics of women who were managed by intrauterine balloon tamponade and uterine artery embolization before and after propensity score-matching are presented in Table 1. Before propensity score-matching, multiple characteristics differed significantly between intervention groups, as indicated by a standardized difference above $10 \%$. Women who were initially managed by intrauterine balloon tamponade were more likely to have a vaginal birth ( $80 \%$ vs $60 \%$ of women in the embolization group) and had less blood loss at the time of balloon insertion (median $2500 \mathrm{~mL}$ [IQR 2000-3000]) compared with women who initially had uterine artery embolization (median $3500 \mathrm{~mL}$ [IQR 3000-4500]). Furthermore, women who initially underwent uterine artery embolization were more likely to have coagulopathy ( $43 \%$ vs $11 \%$ of women in the balloon group), were more often treated with non-uterotonic agents, and received more blood components than women who were managed by intrauterine balloon tamponade. Uterine atony was the leading cause of hemorrhage in both intervention groups. Characteristics were well balanced in the propensity score-matched cohort, with $<10 \%$ standardized differences for all characteristics (Table 1 ).

\section{2 | Comparison of outcomes}

Among the 373 women who initially had intrauterine balloon tamponade, 262 women (70\%) required no additional intervention and bleeding was adequately treated. After intrauterine balloon insertion, 12 women (3\%) had a B-Lynch suture, four women (1\%) had 


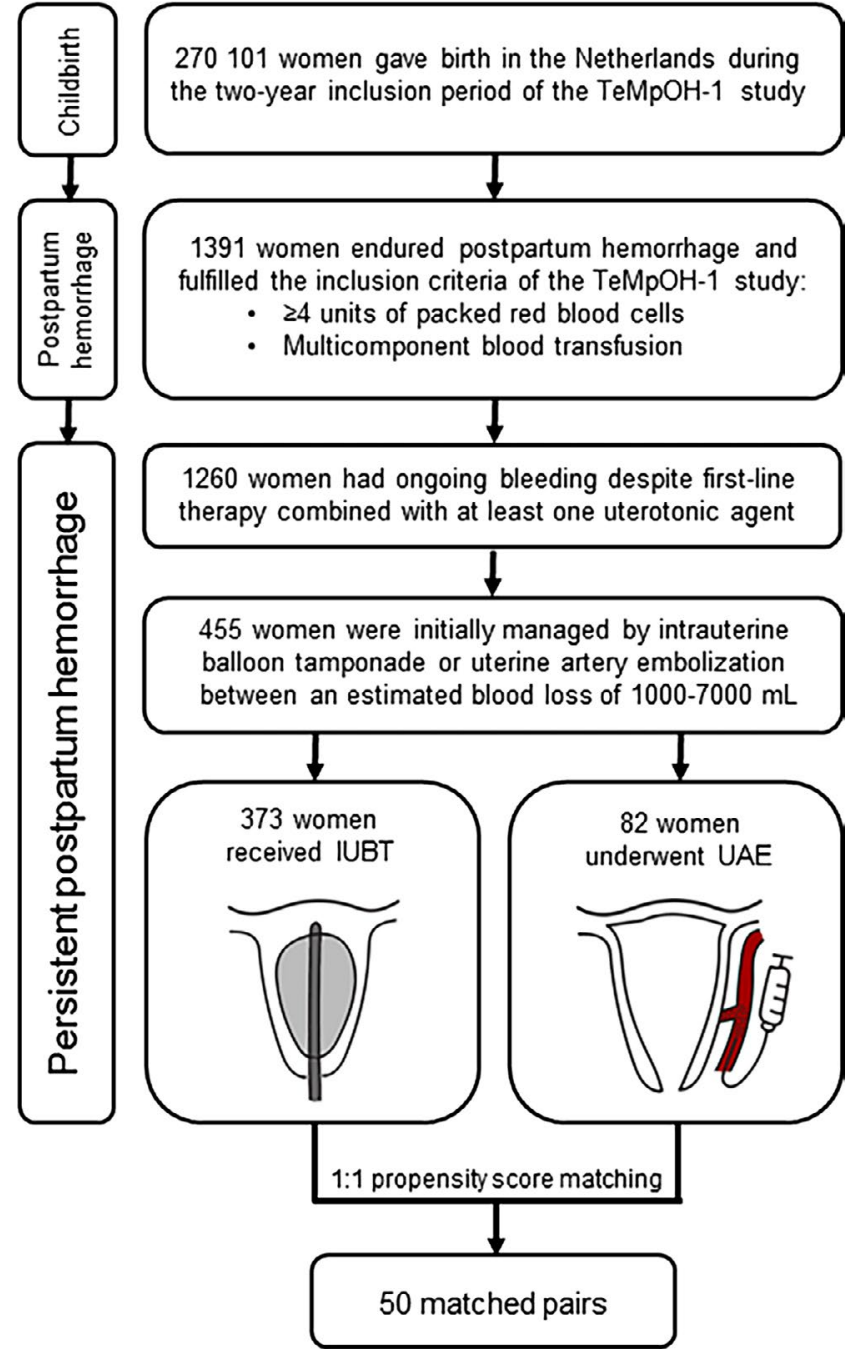

FIGURE 1 Flowchart of study enrollment and propensity scorematching. IUBT, intrauterine balloon tamponade; UAE, uterine artery embolization

uterine artery ligation and 81 women (22\%) still had to undergo uterine artery embolization, 7 of whom eventually underwent hysterectomy. The total number of women who had a peripartum hysterectomy after intrauterine balloon tamponade was 19 (5\%) and 2 women (.5\%) died because of exsanguination before additional interventions could be performed. Of the 82 women who initially underwent uterine artery embolization, 14 women (17\%) endured ongoing hemorrhage, of whom 3 (4\%) had a B-Lynch suture, 1 (1\%) had uterine artery ligation and $10(12 \%)$ required peripartum hysterectomy. None of the women who primarily had uterine artery embolization died. In the unadjusted analysis, the risk of the composite primary outcome (peripartum hysterectomy and or maternal mortality) was higher for women who underwent uterine artery embolization compared with women who received intrauterine balloon tamponade (12\% vs 5.5\% [OR 2.33, 95\% Cl 1.05-5.15]). In addition, total volume of blood loss (median $4500 \mathrm{~mL}$ [IQR 3350-6000] vs $3500 \mathrm{~mL}$ [IQR 3000-4500], respectively, $P<0.001$ ) and total number of packed red blood cells transfused (median 7 units [IQR 5-11] vs 4 units [IQR 3-7], respectively, $P<0.001$ ) were higher for women who underwent uterine artery embolization than for women who had an intrauterine balloon as initial management during persistent postpartum hemorrhage (Table 2). Of all women who had uterine artery embolization (82 as initial management and 81 after intrauterine balloon tamponade), 3 (1.8\%) suffered an embolization-related thrombo-embolic event; 1 of these 3 women had received intrauterine balloon tamponade before uterine artery embolization was applied.

In the propensity score-matched cohort, 29 of the 50 women (58\%) who were initially managed by an intrauterine balloon required no additional intervention to control bleeding. Two women (4\%) had a B-Lynch suture after intrauterine balloon insertion and 15 (30\%) underwent uterine artery embolization after intrauterine balloon tamponade, 2 of whom required peripartum hysterectomy. The total number of women who underwent hysterectomy to arrest hemorrhage was 6 (12\%) both for women who initially had intrauterine balloon tamponade and for women who initially had uterine artery embolization. Maternal deaths occurred in neither of the intervention groups. In the propensity score-matched adjusted analyses, there was no significant difference in the risk of the composite primary outcome between the intervention groups (12\% in each group [OR $1.00,95 \% \mathrm{Cl} .30-3.34]$ ). There was no significant difference either in the total volume of blood loss (median $4500 \mathrm{~mL}$ [IQR $3600-5400$ ] vs $4000 \mathrm{~mL}$ [IQR; 3250-5000], respectively, $P=0.382$ ) or total number of packed red blood cells transfused (median 7 units [IQR 5-10] vs 6 units [IQR 4-9], respectively, $P=0.319$ ) between both who had intrauterine balloon tamponade and women who underwent uterine artery embolization (Table 2). One woman in the propensity score-matched cohort had a thrombo-embolic event related to the embolization performed after initial management with intrauterine balloon tamponade failed.

\section{3 | Sensitivity analysis}

The sensitivity analysis yielded results similar to our primary analysis. When the propensity score was used as the only covariate in the logistic regression model to compare the primary outcome measure between all women who had intrauterine balloon tamponade ( $n=373$ ) and all women who underwent uterine artery embolization ( $n=82$ ) as the initial management for persistent postpartum hemorrhage between an estimated blood loss of $1000-7000 \mathrm{~mL}$, the risk of the composite primary outcome was slightly, but still not statistically significantly, lower among women who were managed by intrauterine balloon tamponade than women who underwent uterine artery embolization (OR .77, 95\% Cl .27-2.21).

\section{4 | DISCUSSION}

This propensity score-matched cohort study found no significant difference in the risk of the composite outcome of peripartum hysterectomy and/or maternal death between women with persistent 
TAB LE 1 Clinically relevant characteristics for women who had intrauterine balloon tamponade or who underwent uterine artery embolization after an estimated blood loss of $1000-7000 \mathrm{~mL}$ because of persistent postpartum hemorrhage before and after propensity score-matching

\begin{tabular}{|c|c|c|c|c|c|c|}
\hline & \multicolumn{3}{|c|}{ Before PS matching } & \multicolumn{2}{|c|}{ After PS matching } & \multirow[b]{2}{*}{ SMD (\%) } \\
\hline & IUBT (n = 373) & UAE (n = 82) & SMD (\%) & IUBT $(n=50)$ & UAE $(n=50)$ & \\
\hline Maternal age, $y^{a}$ & $31(28-35)$ & $32(29-36)$ & 15.6 & $32(29-37)$ & $31(29-36)$ & 5.7 \\
\hline Gestational age, $w^{a}$ & $39(38-40)$ & $38(37-40)$ & 28.9 & $39(37-40)$ & $38(37-40)$ & 6.9 \\
\hline Multiparity, n (\%) & $170(46)$ & $43(52)$ & 16.8 & $28(56)$ & $27(54)$ & 4.0 \\
\hline Preeclampsia, n (\%) & $36(10)$ & $10(12)$ & 7.5 & $6(12)$ & $6(12)$ & .0 \\
\hline Multiple pregnancy, n (\%) & $23(6)$ & $6(7)$ & 7.1 & $4(8)$ & $4(8)$ & .0 \\
\hline Prior cesarean birth, n (\%) & $44(12)$ & $16(20)$ & 22.4 & $9(18)$ & $10(20)$ & 5.0 \\
\hline \multicolumn{7}{|l|}{ Mode of birth, $n(\%)$} \\
\hline Vaginal delivery & $300(80)$ & $49(60)$ & \multirow[t]{2}{*}{54.0} & $28(56)$ & $28(56)$ & \multirow[t]{2}{*}{.0} \\
\hline Cesarean section & $73(20)$ & $33(40)$ & & $22(44)$ & $22(44)$ & \\
\hline \multicolumn{7}{|l|}{ Cause of hemorrhage, $\mathrm{n}(\%)$} \\
\hline Uterine atony & $293(79)$ & $53(64)$ & Ref & $32(64)$ & $33(66)$ & Ref \\
\hline Retained placenta & $45(12)$ & $7(9)$ & 6.6 & $5(10)$ & $5(10)$ & .0 \\
\hline Abnormally invasive placenta & $24(6)$ & $7(9)$ & 19.2 & $6(12)$ & $6(12)$ & .0 \\
\hline Other causes & $11(3)$ & $15(18)$ & 34.7 & $7(14)$ & $6(12)$ & 5.9 \\
\hline Placental abruption & 2 & 1 & - & 2 & 0 & - \\
\hline Placenta previa & 2 & 3 & - & 2 & 1 & - \\
\hline Uterine rupture & 7 & 11 & - & 3 & 5 & - \\
\hline $\begin{array}{l}\text { Estimated blood loss at the time of } \\
\text { the intervention, } \mathrm{mL}^{\mathrm{a}}\end{array}$ & $\begin{array}{l}2500 \\
(2000-3000)\end{array}$ & $\begin{array}{l}3500 \\
(3000-4500)\end{array}$ & 62.5 & $\begin{array}{l}3250 \\
(2500-4000)\end{array}$ & $\begin{array}{l}3250 \\
(2500-4000)\end{array}$ & .0 \\
\hline Coagulopathy, n (\%) & $42(11)$ & $35(43)$ & 60.4 & $18(36)$ & $18(36)$ & .0 \\
\hline Symptoms of shock, n (\%) & $304(82)$ & $68(83)$ & 1.3 & $40(80)$ & $41(82)$ & 5.0 \\
\hline Number of uterotonics given ${ }^{a}$ & $2(2-3)$ & $2(1-3)$ & 28.0 & $2(1-2)$ & $2(1-3)$ & 5.2 \\
\hline \multicolumn{7}{|l|}{ Non-uterotonic agents, n (\%) } \\
\hline Tranexamic acid & $132(35)$ & $42(51)$ & 13.5 & $23(46)$ & $21(42)$ & 8.0 \\
\hline Fibrinogen concentrate & $6(2)$ & $13(16)$ & 30.0 & $4(8)$ & $4(8)$ & .0 \\
\hline Recombinant factor VIla & $3(1)$ & $3(4)$ & 10.1 & $1(2)$ & $1(2)$ & .0 \\
\hline \multicolumn{7}{|l|}{ Blood components $^{a}$} \\
\hline Packed red blood cells & $1(0-2)$ & $4(3-7)$ & 88.7 & $4(2-6)$ & $4(2-5)$ & 9.2 \\
\hline Fresh frozen plasma & $0(0-1)$ & $2(1-4)$ & 80.1 & $2(0-2)$ & $2(0-2)$ & 5.5 \\
\hline Platelet transfusion & $0(0-0)$ & $0(0-1)$ & 48.7 & $0(0-0)$ & $0(0-0)$ & 6.3 \\
\hline Surgical interventions, $\mathrm{n}(\%)$ & $3(1)$ & $2(2)$ & 20.8 & $2(4)$ & $2(4)$ & .0 \\
\hline B-Lynch suture & 3 & 2 & - & 2 & 2 & - \\
\hline Uterine artery ligation & 0 & 1 & - & 0 & 1 & - \\
\hline
\end{tabular}

Abbreviations: IUBT, intrauterine balloon tamponade; PS, propensity score; Ref, reference; SMD, standardized mean difference; UAE, uterine artery embolization.

${ }^{\mathrm{a}}$ Reported as median with (interquartile ranges).

postpartum hemorrhage and women were initially managed by intrauterine balloon tamponade or uterine artery embolization for a blood loss of 1000 and $7000 \mathrm{~mL}$. We also did not find significant differences in total volume of blood loss and total number of packed red blood cells transfused. Thirty-four percent (17/50) of women who were initially managed by intrauterine balloon tamponade had an additional intervention, of whom 15 had uterine artery embolization.
One woman suffered an embolization-related thrombo-embolic event.

To the best of our knowledge this is the first study comparing the effectiveness of intrauterine balloon tamponade with another invasive management strategy to control bleeding and avert peripartum hysterectomy and maternal death during persistent postpartum hemorrhage. By using propensity score-matching, we 
ensured a similar distribution of potential confounding variables between the intervention groups. The definition of persistent postpartum hemorrhage enabled us to overcome differences between caregivers regarding estimation of blood loss and establish a clear point in time at which an additional intervention (ie, intrauterine balloon tamponade or uterine artery embolization) was deemed necessary following failure of initial management. Another key strength is that the composite primary outcome consisted of two postpartum hemorrhage-related core outcome sets (peripartum hysterectomy and maternal death), allowing our results to be potentially included in systematic reviews or metaanalyses on persistent postpartum hemorrhage. ${ }^{31}$ Furthermore, the extensive $\mathrm{TeMpOH}-1$ database made it possible to include many characteristics as potential confounders in the propensity score model. Nonetheless, even though this is the first study that compares the effectiveness of intrauterine balloon tamponade with another invasive management strategy, our propensity score-matched sample size was limited to 50 pairs. This resulted in confidence intervals too broad to rule out type II error for the composite primary outcome measure between the two intervention groups. Limited statistical power also restricted possible comparative analyses of subgroups to determine which characteristics might modify the effect of intrauterine balloon tamponade. However, consistency between the results of our primary analysis and the sensitivity analysis strengthens the credibility of our findings. Nevertheless, our results should be interpreted with caution considering several other limitations in relation to the observational design. We were unable to collect data regarding type of intrauterine balloon device inserted, volume of fluid used to inflate the intrauterine balloon, and the reason for failure of intrauterine balloon tamponade or uterine artery embolization. Additionally, although we are confident that we have included all clinically relevant characteristics associated with the clinical decision to use intrauterine balloon tamponade or uterine artery embolization, residual confounding cannot be ruled out. Finally, women were included when in need of four or more units of packed red blood cells or a multicomponent blood transfusion, with an estimated blood loss of 1000-7000 mL at the time of intervention. Our results can therefore not be generalized to all women who satisfy the criteria for persistent postpartum hemorrhage, but are still applicable to the large majority in settings where both interventions and packed cells are available.

Intrauterine balloon tamponade has been incorporated as a management option into multiple national guidelines for postpartum hemorrhage. ${ }^{32-35}$ In non-comparative studies, success rates of intrauterine balloon tamponade to control bleeding after childbirth varied between $67 \%$ and $91 \% .6,12,13,36$ However, evidence for the benefits of intrauterine balloon tamponade compared with other invasive management strategies is lacking, resulting in uncertainty about whether intrauterine balloon tamponade is effective during the course of persistent postpartum hemorrhage. ${ }^{37}$

Our reported success rate of $70 \%$ among all women who were initially managed by intrauterine balloon tamponade between an 
estimated blood loss of $1000-7000 \mathrm{~mL}$ is in accordance with prior literature. However, the success rate of women who had intrauterine balloon tamponade and who required no additional intervention to control hemorrhage was $58 \%$ in the propensity score-matched cohort. The explanation for this apparent lower success rate could be due to the difference in severity of bleeding. Volume of blood loss at time of intrauterine balloon insertion was lower for the total cohort of women who had intrauterine balloon tamponade (median $2500 \mathrm{~mL}$ [IQR 2000-3000]) than for women in the propensity score-matched cohort (median $3250 \mathrm{~mL}$ [IQR 2500-4000]). This is because we matched women who had intrauterine balloon tamponade with women who had uterine artery embolization within the same increment of blood loss at the time of the intervention. Consequently, there were proportionally more women with intrauterine balloon tamponade in the propensity score-matched cohort who had more severe bleeding than there were in the total cohort of women who were initially managed by intrauterine balloon tamponade. Nevertheless, early timing of intrauterine balloon tamponade during the course of postpartum hemorrhage has been associated with improved maternal outcome, whereas early timing of uterine artery embolization seems to be unrelated to maternal outcome. ${ }^{19,38}$ However, in these studies, early timing of intrauterine balloon tamponade in the absence of a control group could also have led to an overestimation of the effectiveness due to the possibility that the use of intrauterine balloon tamponade was not absolutely necessary.

Although $34 \%$ of women who initially received intrauterine balloon tamponade had an additional intervention, there was no significant difference in the risk of peripartum hysterectomy and or maternal death compared with women who initially underwent uterine artery embolization. Therefore, our results indicate that initial management by intrauterine balloon tamponade during persistent postpartum hemorrhage has the potential to control bleeding and obviate the need for uterine artery embolization in most women, without an increased risk of severe maternal outcome. By using intrauterine balloon tamponade as the intervention of first choice during persistent postpartum hemorrhage, most women can be spared a more invasive and expensive intervention, that is, uterine artery embolization. Two studies corroborate our study findings, reporting a significant drop in the number of invasive procedures after introduction of intrauterine balloon tamponade into their guidelines on management of postpartum hemorrhage after an initial treatment with uterotonic agents failed..$^{39,40}$

However, since our propensity score-matched sample size was small, we can only make cautious statements regarding the effect of both management options on the risk of hysterectomy and or maternal mortality. Furthermore, it is specifically important to note that if uterine artery embolization was not available, it is possible that a larger proportion of women who were initially managed by intrauterine balloon tamponade had peripartum hysterectomy or died. In addition, although intrauterine balloon tamponade seems to be a readily available intervention of first choice in the management of persistent postpartum hemorrhage, it should not delay or be considered a replacement for uterine artery embolization or hysterectomy if that procedure is deemed necessary to control bleeding. On the other hand, intrauterine balloon tamponade could also be used as temporizing measure while awaiting embolization or surgery. ${ }^{41}$

The World Health Organization acknowledges the need for further research into the efficacy of intrauterine balloon tamponade in the management of postpartum hemorrhage. ${ }^{42}$ Considering that uterine artery embolization is not widely available, comparative research on intrauterine balloon tamponade with other management strategies is warranted, particularly in low-resource settings where intrauterine balloon tamponade could be used as cost-saving option to control ongoing bleeding. One randomized trial evaluated the effectiveness of intrauterine balloon tamponade as an adjunct to misoprostol but was underpowered to demonstrate a significant treatment effect. ${ }^{43}$ The inability to resolve the research question of whether intrauterine balloon tamponade is as good as or superior to other management strategies due to small sample sizes, highlights the need for larger studies comparing intrauterine balloon tamponade with other management strategies for a substantiated implementation of intrauterine balloon tamponade into the clinical guidelines for management of postpartum hemorrhage. International research collaboration may be the key to overcome the problem of low statistical power and determine whether and when intrauterine balloon tamponade should be used during the course of postpartum hemorrhage. Our study design provides a useful framework and could serve as a starting point for future comparative effectiveness research of intrauterine balloon tamponade to control intractable postpartum hemorrhage in clinical as well as observational studies.

\section{5 | CONCLUSION}

The risk of the composite outcome of peripartum hysterectomy and/ or maternal death, total volume of blood loss, and total number of packed red blood cells transfused did not significantly differ between women who had intrauterine balloon tamponade and women who had uterine artery embolization as initial management for persistent postpartum hemorrhage. Intrauterine balloon tamponade seems to be a readily available intervention of first choice in the management of persistent postpartum hemorrhage that could obviate the need for uterine artery embolization in most women. However, limited sample size made it difficult to demonstrate equivalence of the two interventions and our results emphasize the need for larger studies comparing intrauterine balloon tamponade with other management options for a substantiated implementation of balloon tamponade into clinical guidelines for management of postpartum hemorrhage.

\section{ACKNOWLEDGEMENTS}

We thank all the 61 participating hospitals and the Dutch consortium for Healthcare Evaluation and Research in Obstetrics and Gynaecology - NVOG Consortium 2.0. We thank all members of the TeMpOH-1 group le Cessie S, Eikenboom HCJ, So-Osman C, van de 
Watering LMG, Zwaginga JJ and Zwart JJ, medical students Loeff RM, van Goeverden RJ, Eijlers B, Hillebrand A, Spelmin SE, Beunder TJ, Harskamp V, Wind M, Koning MD, Cramer RA, Veenstra A, Smith SM and Ensing EE, data managers van Brussel-de Groot CJ, and Zouitni O, and the research nurses Kolster-Bijdevaate C, BourgonjeVerhart MS, Bleeker-Taborh CE and Roos-van Milligen E for their contribution to the TeMpOH-1 study.

\section{CONFLICT OF INTEREST}

Rolf H. H. Groenwold received financial support from the Netherlands Organisation for Scientific Research for the submitted work. No competing interests were reported by the other authors that could appear to have influenced the submitted work.

\section{ORCID}

Paul I. Ramler (iD https://orcid.org/0000-0002-9753-3983

Dacia D. C. A. Henriquez (iD https://orcid.org/0000-0003-3164-8611

Thomas Akker (iD https://orcid.org/0000-0002-9890-9145

Camila Caram-Deelder (iD https://orcid.org/0000-0003-3161-5684

Rolf H. H. Groenwold (ID https://orcid.org/0000-0001-9238-6999

Kitty W. M. Bloemenkamp (iD https://orcid.org/0000-0002-1377-4625

Jos Roosmalen (iD https://orcid.org/0000-0002-6746-3259

Johanna G. Bom iD https://orcid.org/0000-0001-9095-2475

\section{REFERENCES}

1. Say L, Chou D, Gemmill A, et al. Global causes of maternal death: a WHO systematic analysis. Lancet Glob Health. 2014;2(6):e323-e333.

2. Mann S, Hollier LM, McKay K, Brown H. What we can do about maternal mortality - and how to do it quickly. N Engl J Med. 2018;379(18):1689-1691.

3. Zwart JJ, Dijk PD, van Roosmalen J. Peripartum hysterectomy and arterial embolization for major obstetric hemorrhage: a 2-year nationwide cohort study in the Netherlands. Am J Obstet Gynecol. 2010;202(2):150.e1-e7.

4. Knight M, Kurinczuk JJ, Spark P, Brocklehurst P; United Kingdom Obstetric Surveillance System Steering C. Cesarean delivery and peripartum hysterectomy. Obstet Gynecol. 2008;111(1):97-105.

5. van den Akker T, Brobbel C, Dekkers OM, Bloemenkamp KW. Prevalence, indications, risk indicators, and outcomes of emergency peripartum hysterectomy worldwide: a systematic review and meta-analysis. Obstet Gynecol. 2016;128(6):1281-1294.

6. Doumouchtsis SK, Papageorghiou AT, Arulkumaran S. Systematic review of conservative management of postpartum hemorrhage: what to do when medical treatment fails. Obstet Gynecol Surv. 2007;62(8):540-547.

7. Aoki M, Tokue H, Miyazaki M, Shibuya K, Hirasawa S, Oshima K. Primary postpartum hemorrhage: outcome of uterine artery embolization. Br J Radiol. 2018;91(1087):20180132.

8. Ganguli S, Stecker MS, Pyne D, Baum RA, Fan CM. Uterine artery embolization in the treatment of postpartum uterine hemorrhage. $J$ Vasc Interv Radiol. 2011;22(2):169-176.

9. Maassen MS, Lambers MD, Tutein Nolthenius RP, van der Valk $\mathrm{PH}$, Elgersma OE. Complications and failure of uterine artery embolization for intractable postpartum hemorrhage. BJOG. 2009;116(1):55-61.

10. Kirby JM, Kachura JR, Rajan DK, et al. Arterial embolization for primary postpartum hemorrhage. J Vasc Interv Radiol. 2009;20(8):1036-1045.

11. Bakri YN, Amri A, Abdul Jabbar F. Tamponade-balloon for obstetrical bleeding. Int J Gynaecol Obstet. 2001;74(2):139-142.

12. Olsen R, Reisner DP, Benedetti TJ, Dunsmoor-Su RF. Bakri balloon effectiveness for postpartum hemorrhage: a "real world experience". J Matern Fetal Neonatal Med. 2013;26(17):1720-1723.

13. Revert M, Cottenet J, Raynal P, Cibot E, Quantin C, Rozenberg P. Intrauterine balloon tamponade for management of severe postpartum hemorrhage in a perinatal network: a prospective cohort study. BJOG. 2017;124(8):1255-1262.

14. Rosenbaum PR, Rubin DB. The central role of the propensity score in observational studies for causal effects. Biometrika. 1983;70(1):41-55.

15. Gillissen A, Henriquez D, van den Akker T, et al. The effect of tranexamic acid on blood loss and maternal outcome in the treatment of persistent postpartum hemorrhage: a nationwide retrospective cohort study. PLoS ONE. 2017;12(11):e0187555.

16. World Health Organization. Evaluating the Quality of Care for Severe Pregnancy Complications. The WHO Near-miss Approach for Maternal Health. Geneva: WHO; 2011.

17. Brookhart MA, Schneeweiss S, Rothman KJ, Glynn RJ, Avorn J, Sturmer T. Variable selection for propensity score models. Am J Epidemiol. 2006;163(12):1149-1156.

18. Huque S, Roberts I, Fawole B, Chaudhri R, Arulkumaran S, ShakurStill H. Risk factors for peripartum hysterectomy among women with postpartum hemorrhage: analysis of data from the WOMAN trial. BMC Pregnancy Childbirth. 2018;18(1):186.

19. Kong CW, To WW. Prognostic factors for the use of intrauterine balloon tamponade in the management of severe postpartum hemorrhage. Int J Gynaecol Obstet. 2018;142(1):48-53.

20. Cho HY, Park YW, Kim YH, Jung I, Kwon JY. Efficacy of intrauterine Bakri balloon tamponade in cesarean section for placenta previa patients. PLOS ONE. 2015;10(8):e0134282.

21. Lee SM, Shin JH, Shim JJ, et al. Postpartum hemorrhage due to genital tract injury after vaginal delivery: safety and efficacy of transcatheter arterial embolization. Eur Radiol. 2018;28(11):4800-4809.

22. Lee HY, Shin JH, Kim J, et al. Primary postpartum hemorrhage: outcome of pelvic arterial embolization in 251 patients at a single institution. Radiology. 2012;264(3):903-909.

23. Gillissen A, van den Akker T, Caram-Deelder C, et al. Coagulation parameters during the course of severe postpartum hemorrhage: a nationwide retrospective cohort study. Blood Adv. 2018;2(19):2433-2442.

24. Austin PC. Optimal caliper widths for propensity-score matching when estimating differences in means and differences in proportions in observational studies. Pharm Stat. 2011;10(2):150-161.

25. Austin PC. An introduction to propensity score methods for reducing the effects of confounding in observational studies. Multivariate Behav Res. 2011;46(3):399-424.

26. Belitser SV, Martens EP, Pestman WR, Groenwold RH, de Boer A, Klungel $\mathrm{OH}$. Measuring balance and model selection in propensity score methods. Pharmacoepidemiol Drug Saf. 2011;20(11):1115-1129.

27. Kosuke I, King G, Stuart EA. Misunderstanding between experimentalists and observationalists about causal inference. J R Stat Soc A. 2008;171(2):481-502.

28. Sjolander A, Greenland S. Ignoring the matching variables in cohort studies-when is it valid and why? Stat Med. 2013;32(27): 4696-4708.

29. Baek S, Park SH, Won E, Park YR, Kim HJ. Propensity score matching: a conceptual review for radiology researchers. Korean J Radiol. 2015;16(2):286-296 
30. Williamson E, Morley R, Lucas A, Carpenter J. Propensity scores: from naive enthusiasm to intuitive understanding. Stat Methods Med Res. 2012;21(3):273-293.

31. Meher S, Cuthbert A, Kirkham JJ, et al. Core outcome sets for prevention and treatment of postpartum hemorrhage: an international Delphi consensus study. BJOG. 2019;126(1):83-93.

32. Nederlandse Vereniging voor Obstetrie en Gynaecologie. Hemorrhagia postpartum (HPP). Arnhem; 2013. https://www. nvog.nl/wp-content/uploads/2018/02/Hemorrhagia-postpartum-HPP-3.0-14-11-2013.pdf. Accessed January 15, 2019.

33. Sentilhes L, Vayssiere C, Deneux-Tharaux C, et al. Postpartum hemorrhage: guidelines for clinical practice from the French College of Gynaecologists and Obstetricians (CNGOF): in collaboration with the French Society of Anesthesiology and Intensive Care (SFAR). Eur J Obstet Gynecol Reprod Biol. 2016;198:12-21.

34. Mavrides E, Allard S, Chandraharan E, et al.; on behalf of the Royal College of Obstetricians and Gynaecologists. Prevention and Management of Postpartum Hemorrhage: Green-top Guideline No. 52. BJOG. 2017;124(5):e106-e149.

35. Committee on Practice Bulletins-Obstetrics. Practice Bulletin No. 183: Postpartum Hemorrhage. American College of Obstetricians and Gynecologists. Obstet Gynecol. 2017;130(4):e168-e186.

36. Georgiou C. Balloon tamponade in the management of postpartum hemorrhage: a review. BJOG. 2009;116(6):748-757.

37. Viteri OA, Sibai BM. Uterine balloon tamponade for the management of postpartum hemorrhage: a challenge and an opportunity for better evidence. BJOG. 2018;125(5):540.

38. Howard TF, Grobman WA. The relationship between timing of postpartum hemorrhage interventions and adverse outcomes. Am J Obstet Gynecol. 2015;213(2):239.e1-e3.

39. Laas E, Bui C, Popowski T, Mbaku OM, Rozenberg P. Trends in the rate of invasive procedures after the addition of the intrauterine tamponade test to a protocol for management of severe postpartum hemorrhage. Am J Obstet Gynecol. 2012;207(4):281.e1-e7.
40. Gauchotte E, De La Torre M, Perdriolle-Galet E, Lamy C, Gauchotte $\mathrm{G}$, Morel O. Impact of uterine balloon tamponade on the use of invasive procedures in severe postpartum hemorrhage. Acta Obstet Gynecol Scand. 2017;96(7):877-882.

41. Dildy GA, Belfort MA, Adair CD, et al. Initial experience with a dualballoon catheter for the management of postpartum hemorrhage. Am J Obstet Gynecol. 2014;210(2):136.e1-e6.

42. World Health Organization. WHO Recommendations for the Prevention and Treatment of Postpartum Hemorrhage. Geneva: WHO; 2012.

43. Dumont A, Bodin C, Hounkpatin B, et al. Uterine balloon tamponade as an adjunct to misoprostol for the treatment of uncontrolled postpartum hemorrhage: a randomised controlled trial in Benin and Mali. BMJ Open. 2017;7(9):e016590.

\section{SUPPORTING INFORMATION}

Additional supporting information may be found online in the Supporting Information section at the end of the article.

How to cite this article: Ramler PI, Henriquez DDCA, van den Akker T, et al. Comparison of outcome between intrauterine balloon tamponade and uterine artery embolization in the management of persistent postpartum hemorrhage: A propensity score-matched cohort study. Acta Obstet Gynecol Scand. 2019;00:1-10. https://doi.org/10.1111/ aogs.13679 